\title{
Diuretic and Antidiuretic Activities of Ethanol Extract and Fractions of Lagopsis supina in Normal Rats
}

\author{
Zhiyong Liu, ${ }^{1}$ Li Yang $\mathbb{D}^{2}{ }^{2}$ Longxue Li, ${ }^{1}$ Rongrui Wei, ${ }^{3}$ Xiaoquan Luo, ${ }^{1}$ Tingting Xu, ${ }^{3}$ \\ Yun Huang, ${ }^{1}$ Zejing $\mathrm{Mu}^{3}$ and Junwei $\mathrm{He} \mathbb{B}^{3}$ \\ ${ }^{1}$ Laboratory Animal Science and Technology Center, Jiangxi University of Traditional Chinese Medicine, \\ Nanchang 330004, China \\ ${ }^{2}$ Key Laboratory of Modern Preparation of TCM, Ministry of Education, Jiangxi University of Traditional Chinese Medicine, \\ Nanchang 330004, China \\ ${ }^{3}$ Research Center of Natural Resources of Chinese Medicinal Materials and Ethnic Medicine, \\ Jiangxi University of Traditional Chinese Medicine, Nanchang 330004, China
}

Correspondence should be addressed to Junwei He; hjwjn2008@163.com

Received 23 September 2019; Revised 7 November 2019; Accepted 18 November 2019; Published 4 December 2019

Academic Editor: Jane Hanrahan

Copyright (c) 2019 Zhiyong Liu et al. This is an open access article distributed under the Creative Commons Attribution License, which permits unrestricted use, distribution, and reproduction in any medium, provided the original work is properly cited.

Lagopsis supina is a well-known traditional Chinese medicine and used as an agent for diuresis in China for centuries. This is the first time to evaluate the diuretic activity of the ethanol extract of L. supina (LS) and its four fractions (LSA, LSB, LSC, and LSD) in normal rats. After the administration of LS-H, LS-M, LSB-H, and LSC-L, the urine output of the rats was significantly increased, while the urine excretion was significantly reduced after treatment with LSB-L. The urine $\mathrm{Na}^{+}$excretion was remarkably increased with LS-H, LS-M, LSA-H, LSA-L, LSB-H, LSC-L, and LSD-L, and the urine $\mathrm{K}^{+}$excretion was significantly increased after administration of LS-H and LSB-H. Moreover, the urine $\mathrm{Na}^{+}$and $\mathrm{K}^{+}$excretion was significantly reduced after treatment with LSC$\mathrm{H}$ and LSD-H. However, the urine $\mathrm{pH}$ values and urine and serum $\mathrm{Na}^{+}-\mathrm{K}^{+}$-ATPase levels did not show remarkable change after administration of LS or its four fractions in comparison with the control group. On the contrary, LS and its four fractions can suppress the renin-angiotensin-aldosterone system (RAAS), including ADH arrest by LSB-H, LSB-L, LSC-L, LSD-L, and LSD-H and ALD arrest by LSD-L, as well as promote ANP release by LS-M, LSB-H, LSC-H, and LSD-H, while furosemide can suppress only arrest of $\mathrm{ADH}$ within $24 \mathrm{~h}$ compared with the control group. In addition, LS and its four fractions did not change the urine and serum TNF- $\alpha$ and IL- 6 levels in normal rats within $24 \mathrm{~h}$. This study will provide a quantitative basis for explaining the natural medicinal use of LS as a diuretic agent for edema and promoting the diuretic process.

\section{Introduction}

Natural medicine is considered a quite important resource for drug discovery and has increasingly attracted the attention of researchers [1-4]. In Chinese folk medicine, several traditional Chinese medicines (TCMs) are used as diuretic drugs, but most of them lack pharmacological studies to explain their material basis and its mechanisms [5]. Among them, Lagopsis supina (Steph) Ik.-Gal. is a perennial herbaceous plant species of Labiatae family and was widely used as a TCM and known as Xiazhicao (Chinese: 夏至草) [6, 7]. However, due to the indeterminate pharmacological activities and chemical components, $L$. supina still faced big challenges in clinical applications.

Many pharmacological experiments have demonstrated that the crude extract and/or fractions of $L$. supina have promoting blood circulation and removing blood stasis (PBCRBS) [8-10], myocardioprotective [11], and antioxidative [12] effects. Although the pharmacological research of $L$. supina was more, the diuretic activity of LS in normal rats has not yet been studied so far. Furthermore, we found that the ethanol extract of L. supina showed a significant diuretic activity in a rat model of traumatic blood stasis (TBS) [13]. However, it is still unclear whether $L$. 
supina's diuretic activity is caused by PBCRBS constituents and/or diuretic constituents in the TBS rat model. Therefore, there is an urgent need to figure out the material basis and mechanism of diuretic activity of the ethanol extract and its fractions of L. supina in normal rats.

The purpose of the present study was to evaluate the diuretic and antidiuretic activities of the ethanol extract (LS) and its four fractions (LSA, LSB, LSC, and LSD) of L. supina in normal rats within $24 \mathrm{~h}$. For the efficacy evaluation, the urine output volume, urinary electrolyte concentrations $\left(\mathrm{Na}^{+}\right.$and $\left.\mathrm{K}^{+}\right)$, and $\mathrm{pH}$ values were measured at different time points $(1,2,4,6$, and $24 \mathrm{~h})$ after administration, and the $\mathrm{Na}^{+}$$\mathrm{K}^{+}$-ATPase, angiotensin II (Ang II), atriopeptin (ANP), antidiuretic hormone (ADH), aldosterone (ALD), and TNF$\alpha$ and IL- 6 levels in urine and serum were detected within $24 \mathrm{~h}$ after administration.

\section{Materials and Methods}

2.1. Plant Material and Drug Preparation. The plant material $(38.0 \mathrm{~kg})$ and its crude ethanol extract (LS, $8.7 \mathrm{~kg}, 22.9 \%)$ of L. supina were reported in our previous paper, where we followed the methods of $\mathrm{He}$ et al. [13]. In brief, the whole plants of $L$. supina were collected in Keerqin District, Tongliao City, Inner Mongolia, China, in June 2016 having been identified by Prof. Guoyue Zhong (Jiangxi University of Traditional Chinese Medicine). A voucher specimen (no. XZC201606) was deposited at the Research Center of Natural Resources of Chinese Medicinal Materials and Ethnic Medicine, Jiangxi University of Traditional Chinese Medicine, Nanchang, China. Then, the air-dried and powdered whole plants $(38 \mathrm{~kg})$ were exhaustively extracted using $95 \% \mathrm{EtOH}(300 \mathrm{~L} \times 3)$ and subsequently $50 \% \mathrm{EtOH}$ $(300 \mathrm{~L} \times 3)$ by maceration at room temperature for seven days. After filtration, combination, and solvent evaporation, the crude ethanol extract of L. supina (LS, $8.7 \mathrm{~kg}, 22.90 \%$ ) was obtained. Then, LS was suspended in water and extracted with hexane, to afford the hexane portion (LSA, $1320 \mathrm{~g}$ ) and water portion, respectively. The water portion was chromatographed on a D101 macroporous resin column and eluted with $0 \%, 30 \%, 60 \%$, and $95 \%$ aqueous $\mathrm{EtOH}$ to yield four fractions: $0 \%$ (LSB, $6148 \mathrm{~g}, 16.18 \%$ ), 30\% (LSC, $669 \mathrm{~g}, 1.76 \%$ ), 60\% (LSD, $260 \mathrm{~g}, 0.68 \%$ ), and 95\% (LSE, $145 \mathrm{~g})$. The LSA and LSE fractions were combined by highperformance liquid chromatography (HPLC) and thin layer chromatography (TLC) analysis and assigned to LSA $(1465 \mathrm{~g}, 3.86 \%)$. The crude ethanol extract and four fractions were resuspended in $0.3 \%$ sodium carboxymethyl cellulose (CMC-Na) for subsequent intragastric administration and kept at $-20^{\circ} \mathrm{C}$ until use.

According to clinical practice of TCM [6], the dosage of L. supina for rats is $1 \sim 3 \mathrm{~g} / \mathrm{kg} / \mathrm{day}$, which was reported in our previous paper [13]. In this experiment, the yield of LS was $22.90 \%$, making the dosage of the LS crude extract about $229.0 \mathrm{mg} / \mathrm{kg} /$ day.

2.2. Chemicals and Reagents. Enzyme-linked immunosorbent assay (ELISA) kits for $\mathrm{Na}^{+}-\mathrm{K}^{+}$-ATPase, Ang II, ANP,
ADH, ALD, TNF- $\alpha$, and IL- 6 were purchased from Nanjing SenBeiJia Biological Technology Co., Ltd. (Nanjing, China). The levels of urinary $\mathrm{Na}^{+}$and $\mathrm{K}^{+}$were quantified by an automatic biochemical analyzer (OLYMPUS 5421, Olympus Corporation, Japan). All of the other chemicals used in this study were of analytical reagent grade.

2.3. Reference Drug. Furosemide (Jiangsu Yabang Aipusen Pharmaceutical Corporation, Jiangsu, China) was used as a reference drug, which was a high-ceiling loop diuretic in clinical practice, and dissolved in water prior to administration.

2.4. Animal Treatment and Sample Collection. Male SpragueDawley rats weighing $180-220 \mathrm{~g}$ were purchased from Hunan SJA Laboratory Animals Co., Ltd. (Changsha, China). All rats were housed in a temperature-controlled room $\left((23 \pm 2)^{\circ} \mathrm{C}\right)$ with $12 \mathrm{~h}$ light and dark cycles and were allowed free access to food and water ad libitum. All animal procedures were approved by the Institutional Animal Care and Use Committee of Jiangxi University of Traditional Chinese Medicine.

The diuretic activity was determined according to the method published previously $[14,15]$. All rats were randomly assigned into 13 groups ( $n=8$ per group): (1) control group (water-loaded rats), (2) furosemide group: $20 \mathrm{mg} / \mathrm{kg}$ body weight (BW) of furosemide, (3) LS-L group: $114.5 \mathrm{mg} /$ $\mathrm{kg} \mathrm{BW}$ of LS, (4) LS-M group: $229.0 \mathrm{mg} / \mathrm{kg} \mathrm{BW}$ of LS, (5) LSH group: $458.0 \mathrm{mg} / \mathrm{kg}$ BW of LS, (6) LSA-L group: $19.3 \mathrm{mg} /$ $\mathrm{kg} \mathrm{BW}$ of LSA, (7) LSA-H group: $77.2 \mathrm{mg} / \mathrm{kg} \mathrm{BW}$ of LSA, (8) LSB-L group: $80.9 \mathrm{mg} / \mathrm{kg} \mathrm{BW}$ of LSB, (9) LSB-H group: $323.6 \mathrm{mg} / \mathrm{kg} \mathrm{BW}$ of LSB, (10) LSC-L group: $8.8 \mathrm{mg} / \mathrm{kg} \mathrm{BW}$ of LSC, (11) LSC-H group: $35.2 \mathrm{mg} / \mathrm{kg}$ BW of LSC, (12) LSD-L group: $3.4 \mathrm{mg} / \mathrm{kg} \mathrm{BW}$ of LSD, and (13) LSD-H group: $13.6 \mathrm{mg} / \mathrm{kg} \mathrm{BW}$ of LSD. And each group was given the corresponding drug, and the control group received an equivalent amount of water with $0.3 \%$ CMC-Na. Each rat was housed individually in a metabolic cage, and the cumulative urine output was measured at $1,2,4,6$, and $24 \mathrm{~h}$ after administration. Urinary electrolyte concentrations $\left(\mathrm{Na}^{+}\right.$and $\left.\mathrm{K}^{+}\right)$and $\mathrm{pH}$ values were determined in the $24 \mathrm{~h}$ urine samples of all rats. The blood samples were collected from the abdominal aorta of rats and subsequently subjected to centrifugation $\left(3000 \mathrm{rpm}\right.$ at $4^{\circ} \mathrm{C}$ for $10 \mathrm{~min}$ ). The urine and serum were stored at -20 and $-80^{\circ} \mathrm{C}$ for further analysis, respectively.

2.5. Biochemical Methods. Levels of $\mathrm{Na}^{+}-\mathrm{K}^{+}$-ATPase, Ang II, ANP, ADH, ALD, TNF- $\alpha$, and IL- 6 in urine and serum were determined using commercial ELISA kits (Nanjing SenBeiJia Biological Technology Co., Ltd., Nanjing, China), which were performed as per the manufacturer's instructions.

2.6. Statistical Analysis. Data were analyzed using SPSS Statistics V17.0 software and presented as mean \pm standard deviation (SD). The differences between the groups were assessed by a one-way analysis of variance (ANOVA) and 
Tukey's test. Differences with $P<0.05$ indicated statistical significance.

\section{Results}

3.1. Effects on Urinary Excretion Volume. To determine whether the ethanol extract and its four fractions (LSA, LSB, LSC, and LSD) of LS have the diuretic effects, we measured the urinary excretion volume in every group at different time points $(1,2,4,6$, and $24 \mathrm{~h})$ after treatment with LS and its four fractions or furosemide. The urine output volume increased significantly $(P<0.05$ or $P<0.01)$ for LSA-L $(19.3 \mathrm{mg} / \mathrm{kg})$, LS-L $(114.5 \mathrm{mg} / \mathrm{kg})$, and LS-M $(229.0 \mathrm{mg} / \mathrm{kg})$ within 1,2 , and $4 \mathrm{~h}$, respectively, while LSD-H $(13.6 \mathrm{mg} / \mathrm{kg})$ produced a remarkable reduction in the urinary excretion volume within $4 \mathrm{~h}$ after administration compared with the control group $(P<0.05$, Table 1$)$. In addition, urinary excretion volume in LS-H (458.0 mg $/ \mathrm{kg})$, LS-M $(229.0 \mathrm{mg} / \mathrm{kg})$, LSB-H $(322.6 \mathrm{mg} / \mathrm{kg})$, and LSC-L $(8.8 \mathrm{mg} / \mathrm{kg})$ groups significantly increased within $24 \mathrm{~h}$ after administration $(P<0.05$ or $P<0.01)$, while LSB-L $(80.9 \mathrm{mg} / \mathrm{kg})$ produced a remarkable reduction in the urinary excretion volume within $24 \mathrm{~h}$ after administration $(P<0.01$, Table 1$)$. Actually, LSA-H $(77.2 \mathrm{mg} / \mathrm{kg})$, LSC-H $(35.2 \mathrm{mg} / \mathrm{kg})$, LSD-H $(13.6 \mathrm{mg} /$ $\mathrm{kg})$, and LSD-L ( $3.4 \mathrm{mg} / \mathrm{kg}$ ) also contributed to urine output volume, and the increase did not reach statistical significance within $24 \mathrm{~h}$ after administration.

Compared with that in the control group, the urine output volume in the furosemide group significantly increased within $1,2,4,6$, and $24 \mathrm{~h}$ after administration $(P<0.01$, Table 1$)$. Moreover, the urine output volume in the furosemide group was significantly increased compared with that in other groups within $1,2,4$, and $6 \mathrm{~h}$ after administration $(P<0.01$, Table 1$)$; however, the cumulative urine output volume in the furosemide group and LSB-H group within $24 \mathrm{~h}$ showed no significant difference (Table 1).

3.2. Effects on Urinary pH. As shown in Table 2, LS and its four fractions did not change the urine $\mathrm{pH}$ in normal rats within $24 \mathrm{~h}$ in comparison with the control group. The results indicated that LS and its four fractions might play major roles in acid-basic balances in normal rats within $24 \mathrm{~h}$.

\subsection{Effects on Urinary Electrolyte Concentrations. We also} examined urine $\mathrm{Na}^{+}$and $\mathrm{K}^{+}$concentrations in every group at different time points $(1,2,4,6$, and $24 \mathrm{~h})$ after treatment with LS and its four fractions or furosemide (Tables 3 and 4).

Compared with that in the normal group, the urine $\mathrm{Na}^{+}$ concentration in the furosemide treatment group was significantly reduced within $4 \mathrm{~h}$, while the $\mathrm{Na}^{+}$concentration was significantly increased within $24 \mathrm{~h}(P<0.05$ or $P<0.01$, Table 3$)$. In contrast, the urine $\mathrm{K}^{+}$concentration was remarkably increased in the furosemide group within $6 \mathrm{~h}$, while the $\mathrm{K}^{+}$concentration was dramatically reduced within $24 \mathrm{~h}(P<0.05$ or $P<0.01$, Table 4$)$.

After the oral administration of LS and its four fractions within $24 \mathrm{~h}$, a great variation was also observed in urinary electrolyte $\mathrm{Na}^{+}$and $\mathrm{K}^{+}$concentrations. Administration of
LS-H (458.0 mg/kg), LS-M (229.0 mg/kg), LSA-H (77.2 mg/ $\mathrm{kg})$, LSA-L $(19.3 \mathrm{mg} / \mathrm{kg}), \quad$ LSB-H $(322.6 \mathrm{mg} / \mathrm{kg})$, LSC-L $(8.8 \mathrm{mg} / \mathrm{kg})$, and LSD-L $(3.4 \mathrm{mg} / \mathrm{kg})$ significantly increased the urinary electrolyte $\mathrm{Na}^{+}$concentrations within $24 \mathrm{~h}$ $(P<0.05$ or $P<0.01)$, while LSC-H $(35.2 \mathrm{mg} / \mathrm{kg})$ and LSD-H $(13.6 \mathrm{mg} / \mathrm{kg})$ produced a remarkable reduction in the urinary electrolyte $\mathrm{Na}^{+}$concentrations within $24 \mathrm{~h}(P<0.05$ or $P<0.01$, Table 3). Furthermore, the levels of $\mathrm{K}^{+}$concentrations were remarkably reduced after treatment with oral administration of LSC-H $(35.2 \mathrm{mg} / \mathrm{kg})$ and LSD-H $(13.6 \mathrm{mg} /$ $\mathrm{kg})$, while LS-H $(458.0 \mathrm{mg} / \mathrm{kg})$ and LSB-H $(323.6 \mathrm{mg} / \mathrm{kg})$ significantly increased the urinary electrolyte $\mathrm{K}^{+}$concentrations within $24 \mathrm{~h}(P<0.05$ or $P<0.01$, Table 4$)$.

3.4. Effects on Urine and Serum Levels of $\mathrm{Na}^{+}-\mathrm{K}^{+}$-ATPase. Since conventional diuretics can induce electrolyte changes in urine and serum, we examined the levels of $\mathrm{Na}^{+}-\mathrm{K}^{+}$ATPase in urine and serum of normal rats and drug-treated rats. However, administration of LS and its four fractions did not significantly change the urine and serum $\mathrm{Na}^{+}-\mathrm{K}^{+}$ATPase levels in normal rats within $24 \mathrm{~h}$ in comparison with the control group (Table 5).

3.5. Effects on Urine and Serum Levels of Ang II, ANP, ADH, and $A L D$. As shown in Tables 6 and 7, the urine levels of $\mathrm{ADH}$ were remarkably reduced after treatment with oral administration of furosemide within $24 \mathrm{~h}(P<0.01)$. With the treatment of LS-M $(229.0 \mathrm{mg} / \mathrm{kg})$, the levels of ANP in urine were significantly increased within $24 \mathrm{~h}(P<0.05)$.

Interestingly, the four fractions of LS remarkably reduced ADH and ALD levels and significantly increased ANP levels in urine and/or serum compared with control group rats within $24 \mathrm{~h}(P<0.05$ or $P<0.01$, Tables 6 and 7$)$. Treatment with LSB tended to increase ANP levels and to reduce $\mathrm{ADH}$ levels in urine compared with control group rats within $24 \mathrm{~h}(P<0.01)$. Moreover, the levels of ADH reduced and those of ANP increased in urine and/or serum significantly after treatment with LSC compared with control group rats within $24 \mathrm{~h}(P<0.05$ or $P<0.01)$. In addition, we also found that the levels of ADH and ALD were reduced and ANP levels were increased in urine and/or serum significantly after treatment with LSD compared with control group rats within $24 \mathrm{~h}(P<0.05$ or $P<0.01)$.

The result indicates that LS and its four fractions can promote ANP release and suppress the RAAS, including the arrest of ADH and ALD, while furosemide can suppress only arrest of $\mathrm{ADH}$. Thus, as a diuretic, LS and its fractions are superior to furosemide based on multiple components, multiple channels, and multiple targets to treat diuretic diseases.

3.6. Effects on Urine and Serum Levels of TNF- $\alpha$ and IL-6. Inflammation is a complex biological response of the innate immune system and produces a number of proinflammatory mediators, such as TNF- $\alpha$, IL-6, and others. The levels of TNF- $\alpha$ and IL- 6 in urine and serum were measured to assess the effect of LS and its four fractions on inflammation by 
TABLE 1: Effects of LS, LSA, LSB, LSC, and LSD on urinary excretion volume (mL) within 1, 2, 4, 6, and $24 \mathrm{~h}$ after oral administration.

\begin{tabular}{|c|c|c|c|c|c|}
\hline Group & $1 \mathrm{~h}$ & $2 \mathrm{~h}$ & $4 \mathrm{~h}$ & $6 \mathrm{~h}$ & $24 \mathrm{~h}$ \\
\hline Control & $2.07 \pm 0.64$ & $4.45 \pm 1.28$ & $6.77 \pm 1.23$ & $9.13 \pm 1.25$ & $17.73 \pm 0.80$ \\
\hline Furosemide & $7.15 \pm 2.00^{* *}$ & $11.45 \pm 1.69^{* *}$ & $14.02 \pm 2.11^{* *}$ & $16.85 \pm 1.89^{* *}$ & $34.68 \pm 3.51^{* *}$ \\
\hline LS-H & $2.37 \pm 1.00$ & $4.53 \pm 2.08$ & $6.58 \pm 2.22$ & $9.72 \pm 2.28$ & $28.27 \pm 3.89^{* *}$ \\
\hline LS-M & $3.28 \pm 1.64$ & $6.22 \pm 1.97$ & $8.87 \pm 1.65^{*}$ & $10.63 \pm 1.79$ & $25.30 \pm 2.71^{* *}$ \\
\hline LS-L & $2.98 \pm 1.05$ & $6.52 \pm 1.63^{*}$ & $8.18 \pm 1.60$ & $9.43 \pm 1.56$ & $17.17 \pm 1.95$ \\
\hline LSA-H & $3.50 \pm 1.47$ & $6.27 \pm 2.21$ & $8.27 \pm 2.08$ & $10.53 \pm 2.30$ & $24.68 \pm 2.50$ \\
\hline LSA-L & $3.98 \pm 0.89^{* *}$ & $5.80 \pm 1.45$ & $7.37 \pm 1.75$ & $9.45 \pm 1.98$ & $19.75 \pm 2.07$ \\
\hline LSB-H & $2.78 \pm 1.36$ & $5.30 \pm 1.38$ & $7.82 \pm 1.60$ & $10.98 \pm 2.08$ & $38.18 \pm 3.45^{* *}$ \\
\hline LSB-L & $3.12 \pm 0.99$ & $5.18 \pm 1.37$ & $6.63 \pm 1.76$ & $8.68 \pm 1.02$ & $15.13 \pm 1.97^{* *}$ \\
\hline LSC-H & $2.00 \pm 0.93$ & $5.00 \pm 1.74$ & $6.14 \pm 2.01$ & $8.10 \pm 1.07$ & $24.62 \pm 3.07$ \\
\hline LSC-L & $2.98 \pm 0.81$ & $5.52 \pm 0.96$ & $7.83 \pm 1.97$ & $12.27 \pm 1.41$ & $27.25 \pm 3.00^{* *}$ \\
\hline LSD-H & $2.25 \pm 1.00$ & $3.77 \pm 1.23$ & $5.10 \pm 1.11^{*}$ & $7.33 \pm 1.81$ & $20.73 \pm 2.80$ \\
\hline LSD-L & $1.19 \pm 0.61$ & $4.08 \pm 0.90$ & $6.58 \pm 1.41$ & $9.23 \pm 1.74$ & $21.02 \pm 2.38$ \\
\hline
\end{tabular}

The cumulative values are reported as mean \pm SD for 8 rats in each group. ${ }^{*} P<0.05$ and ${ }^{* *} P<0.01$ compared to the control.

TABLE 2: Effects of LS, LSA, LSB, LSC, and LSD on urinary $\mathrm{pH}$ within $1,2,4,6$, and $24 \mathrm{~h}$ after oral administration.

\begin{tabular}{lcccrr}
\hline Group & $1 \mathrm{~h}$ & $2 \mathrm{~h}$ & $4 \mathrm{~h}$ & $6 \mathrm{~h}$ & $24 \mathrm{~h}$ \\
\hline Control & $7.48 \pm 0.46$ & $7.39 \pm 0.54$ & $7.71 \pm 0.19$ & $7.79 \pm 0.16$ & $7.58 \pm 0.72$ \\
Furosemide & $7.66 \pm 0.66$ & $7.59 \pm 0.67$ & $7.47 \pm 0.50$ & $7.21 \pm 0.74$ & $7.69 \pm 0.84$ \\
LS-H & $7.67 \pm 0.43$ & $7.65 \pm 0.35$ & $7.43 \pm 0.48$ & $7.68 \pm 0.48$ & 0.91 \\
LS-M & $7.98 \pm 0.49$ & $7.68 \pm 0.75$ & $7.77 \pm 0.72$ & $7.88 \pm 0.56$ & $8.04 \pm 0.63$ \\
LS-L & $7.86 \pm 0.28$ & $7.83 \pm 0.43$ & $7.73 \pm 0.68$ & $7.43 \pm 0.78$ & $8.18 \pm 0.50$ \\
LSA-H & $7.64 \pm 0.63$ & $7.74 \pm 0.62$ & $7.57 \pm 0.61$ & $7.41 \pm 0.50$ & $8.13 \pm 0.51$ \\
LSA-L & $7.81 \pm 0.42$ & $7.57 \pm 0.46$ & $7.31 \pm 0.33$ & $7.64 \pm 0.54$ & $8.48 \pm 0.65$ \\
LSB-H & $7.74 \pm 0.38$ & $7.60 \pm 0.37$ & $7.58 \pm 0.30$ & $7.58 \pm 0.51$ & $7.97 \pm 0.24$ \\
LSB-L & $7.83 \pm 0.40$ & $7.78 \pm 0.38$ & $7.76 \pm 0.63$ & $7.58 \pm 0.51$ & $8.16 \pm 0.37$ \\
LSC-H & $7.68 \pm 0.27$ & $7.50 \pm 0.51$ & $7.54 \pm 0.59$ & $7.80 \pm 0.85$ & $8.26 \pm 0.57$ \\
LSC-L & $7.72 \pm 0.56$ & $7.63 \pm 0.51$ & $7.94 \pm 0.54$ & $7.08 \pm 0.44$ & $8.25 \pm 0.61$ \\
LSD-H & $7.64 \pm 0.64$ & $7.81 \pm 0.70$ & $7.35 \pm 0.35$ & \\
LSD-L & $7.83 \pm 0.45$ & $7.69 \pm 0.46$ &
\end{tabular}

The values are reported as mean \pm SD for 8 rats in each group.

TABLE 3: Effects of LS, LSA, LSB, LSC, and LSD on urinary electrolyte $\mathrm{Na}^{+}$(mmol/L) concentrations within $1,2,4,6$, and $24 \mathrm{~h}$ after oral administration.

\begin{tabular}{lccccc}
\hline Group & $1 \mathrm{~h}$ & $2 \mathrm{~h}$ & $4 \mathrm{~h}$ & $6 \mathrm{~h}$ & $24 \mathrm{~h}$ \\
\hline Control & $101.88 \pm 7.77$ & $100.38 \pm 8.50$ & $99.33 \pm 9.33$ & $100.18 \pm 2.04$ & $100.30 \pm 3.87$ \\
Furosemide & $59.75 \pm 1.34^{* *}$ & $67.43 \pm 8.02^{* *}$ & $84.65 \pm 3.77^{*}$ & $96.00 \pm 5.53$ & $111.80 \pm 8.59^{*}$ \\
LS-H & $111.80 \pm 8.59$ & $114.13 \pm 8.26$ & $117.45 \pm 8.13^{*}$ & $120.15 \pm 7.29^{* *}$ & $119.80 \pm 8.69^{* *}$ \\
LS-M & $101.55 \pm 11.09$ & $102.28 \pm 9.82$ & $107.48 \pm 9.73$ & $114.15 \pm 11.83$ & $120.68 \pm 1.78^{* *}$ \\
LS-L & $118.20 \pm 6.20^{*}$ & $117.30 \pm 7.47^{*}$ & $116.58 \pm 6.93^{*}$ & $115.83 \pm 6.51^{* *}$ & $108.20 \pm 10.16$ \\
LSA-H & $114.95 \pm 18.99$ & $116.13 \pm 14.65$ & $121.00 \pm 17.08$ & $125.00 \pm 12.53$ & $142.28 \pm 18.97^{* *}$ \\
LSA-L & $75.98 \pm 7.60^{*}$ & $78.00 \pm 4.45^{*}$ & $91.18 \pm 6.00$ & $100.28 \pm 6.75$ & $121.70 \pm 9.37^{* *}$ \\
LSB-H & $128.18 \pm 2.54^{* *}$ & $124.05 \pm 3.26^{* *}$ & $111.65 \pm 5.31$ & $107.83 \pm 4.08^{*}$ & $111.55 \pm 4.57^{* *}$ \\
LSB-L & $115.78 \pm 9.47$ & $116.95 \pm 17.14$ & $109.33 \pm 15.01$ & $102.30 \pm 11.55$ & $91.00 \pm 7.95$ \\
LSC-H & $94.95 \pm 4.84$ & $94.90 \pm 5.96$ & $85.10 \pm 7.54$ & $83.05 \pm 9.22^{*}$ & $78.38 \pm 8.23^{* *}$ \\
LSC-L & $114.73 \pm 9.48$ & $110.80 \pm 7.39$ & $111.38 \pm 11.17$ & $112.35 \pm 6.18^{* *}$ & $126.13 \pm 10.13^{* *}$ \\
LSD-H & $106.03 \pm 7.42$ & $98.60 \pm 4.03$ & $94.50 \pm 3.89$ & $88.10 \pm 4.62^{* *}$ & $74.43 \pm 7.09^{* *}$ \\
LSD-L & $115.55 \pm 5.26^{*}$ & $113.10 \pm 4.52^{*}$ & $107.33 \pm 5.44$ & $112.08 \pm 2.39^{* *}$ & $118.63 \pm 5.80^{* *}$ \\
\hline
\end{tabular}

The values are reported as mean \pm SD for 8 rats in each group. ${ }^{*} P<0.05$ and ${ }^{* *} P<0.01$ compared to the control.

respective ELISA kits. As shown in Table 8, the ELISA results revealed that administration of LS and its four fractions did not affect the urine and serum TNF- $\alpha$ and IL- 6 levels in normal rats within $24 \mathrm{~h}$ in comparison with the control group.

\section{Discussion}

The urinary excretion volume was mainly determined by the urine concentrating ability, including urine and its electrolyte reabsorption $[13,16]$. In this study, we explored the 
TABLE 4: Effects of LS, LSA, LSB, LSC, and LSD on urinary electrolyte $\mathrm{K}^{+}$(mmol/L) concentrations within $1,2,4,6$, and $24 \mathrm{~h}$ after oral administration.

\begin{tabular}{|c|c|c|c|c|c|}
\hline Group & $1 \mathrm{~h}$ & $2 \mathrm{~h}$ & $4 \mathrm{~h}$ & $6 \mathrm{~h}$ & $24 \mathrm{~h}$ \\
\hline Control & $78.98 \pm 5.35$ & $79.40 \pm 5.53$ & $79.38 \pm 5.41$ & $78.83 \pm 5.08$ & $79.03 \pm 5.38$ \\
\hline Furosemide & $150.85 \pm 26.84^{* *}$ & $148.80 \pm 24.93^{* *}$ & $122.00 \pm 10.53^{* *}$ & $95.20 \pm 1.40^{* *}$ & $61.55 \pm 10.50^{*}$ \\
\hline LS-H & $97.80 \pm 2.37^{* *}$ & $96.88 \pm 2.29^{* *}$ & $95.35 \pm 2.03^{* *}$ & $94.85 \pm 2.33^{*}$ & $94.48 \pm 2.92^{* *}$ \\
\hline LS-M & $93.83 \pm 6.13^{*}$ & $91.28 \pm 5.01^{*}$ & $85.73 \pm 4.81$ & $84.00 \pm 5.36$ & $85.38 \pm 4.60$ \\
\hline LS-L & $86.80 \pm 6.34$ & $85.45 \pm 6.12$ & $85.43 \pm 3.75$ & $84.00 \pm 3.41$ & $74.88 \pm 6.97$ \\
\hline LSA-H & $62.18 \pm 3.20^{* *}$ & $58.20 \pm 3.40^{* *}$ & $57.30 \pm 2.08^{* *}$ & $61.25 \pm 0.94^{* *}$ & $77.50 \pm 5.12$ \\
\hline LSA-L & $76.28 \pm 7.20$ & $75.83 \pm 6.19$ & $77.58 \pm 4.64$ & $77.75 \pm 4.48$ & $76.75 \pm 2.86$ \\
\hline LSB-H & $79.75 \pm 2.41$ & $81.08 \pm 1.86$ & $81.98 \pm 2.75$ & $82.20 \pm 2.82$ & $90.28 \pm 5.46^{*}$ \\
\hline LSB-L & $65.50 \pm 11.12$ & $68.33 \pm 7.23$ & $68.58 \pm 7.01$ & $68.83 \pm 7.47$ & $71.58 \pm 8.31$ \\
\hline LSC-H & $70.43 \pm 3.19^{*}$ & $69.53 \pm 2.28^{*}$ & $69.63 \pm 1.79^{*}$ & $67.68 \pm 2.34^{* *}$ & $58.78 \pm 5.93^{* *}$ \\
\hline LSC-L & $82.15 \pm 10.62$ & $81.33 \pm 10.07$ & $79.80 \pm 9.77$ & $74.53 \pm 8.26$ & $75.80 \pm 10.62$ \\
\hline LSD-H & $65.25 \pm 5.55^{*}$ & $63.38 \pm 5.58^{* *}$ & $61.85 \pm 4.26^{* *}$ & $60.58 \pm 4.03^{* *}$ & $52.13 \pm 6.36^{* *}$ \\
\hline LSD-L & $85.78 \pm 3.46$ & $83.23 \pm 2.10$ & $81.28 \pm 2.99$ & $80.48 \pm 1.50$ & $78.30 \pm 1.26$ \\
\hline
\end{tabular}

The values are reported as mean \pm SD for 8 rats in each group. ${ }^{*} P<0.05$ and ${ }^{* *} P<0.01$ compared to the control.

TABLE 5: Effects of LS, LSA, LSB, LSC, and LSD on the levels of $\mathrm{Na}^{+}-\mathrm{K}^{+}$-ATPase (U/L) in rat urine and serum within 24 hours after oral administration.

\begin{tabular}{lcr}
\hline Group & Urine & Serum \\
\hline Control & $92.95 \pm 16.67$ & $105.21 \pm 16.41$ \\
Furosemide & $100.3 \pm 16.10$ & $107.42 \pm 13.66$ \\
LS-H & $99.35 \pm 19.76$ & $90.41 \pm 15.82$ \\
LS-M & $116.4 \pm 17.37$ & $90.72 \pm 10.28$ \\
LS-L & $87.29 \pm 14.46$ & $99.99 \pm 10.65$ \\
LSA-H & $99.71 \pm 15.73$ & $89.25 \pm 16.40$ \\
LSA-L & $95.68 \pm 18.08$ & $91.72 \pm 14.58$ \\
LSB-H & $94.54 \pm 12.40$ & $98.32 \pm 19.97$ \\
LSB-L & $106.55 \pm 11.51$ & $102.66 \pm 15.34$ \\
LSC-H & $114.59 \pm 9.68$ & $116.97 \pm 17.48$ \\
LSC-L & $114.93 \pm 15.05$ & $96.21 \pm 19.63$ \\
LSD-H & $112.65 \pm 15.58$ & $87.12 \pm 10.88$ \\
LSD-L & $102.82 \pm 13.12$ & $106.06 \pm 13.50$ \\
\hline
\end{tabular}

The values are reported as mean \pm SD for 8 rats in each group.

TABLE 6: Effects of LS, LSA, LSB, LSC, and LSD on the levels of Ang II, ANP, ADH, and ALD (pg/mL) in rat urine within $24 \mathrm{~h}$ after oral administration.

\begin{tabular}{lcccc}
\hline Group & Ang II & ANP & ADH & ALD \\
\hline Control & $215.7 \pm 16.76$ & $199.69 \pm 13.48$ & $24.46 \pm 1.42$ & $236.03 \pm 16.08$ \\
Furosemide & $197.30 \pm 14.71$ & $223.41 \pm 18.81$ & $18.42 \pm 2.57^{* *}$ & $205.60 \pm 12.87$ \\
LS-H & $206.79 \pm 18.88$ & $229.88 \pm 11.52$ & $22.75 \pm 2.04$ & $200.7 \pm 15.40$ \\
LS-M & $246.53 \pm 18.14$ & $199.61 \pm 14.38$ & $21.16 \pm 1.66$ & $205.56 \pm 14.94$ \\
LS-L & $218.32 \pm 11.81$ & $188.30 \pm 17.64$ & $24.69 \pm 1.50$ & $214.25 \pm 14.94$ \\
LSA-H & $238.72 \pm 16.79$ & $227.79 \pm 19.81$ & $23.12 \pm 1.92$ & $188.04 \pm 13.13$ \\
LSA-L & $201.67 \pm 10.33$ & $208.45 \pm 15.71$ & $23.66 \pm 1.88$ & $211.11 \pm 17.39$ \\
LSB-H & $176.96 \pm 10.49$ & $234.5 \pm 8.07^{* *}$ & $20.74 \pm 1.97^{* *}$ & $254.52 \pm 13.87$ \\
LSB-L & $218.62 \pm 17.33$ & $207.66 \pm 10.41$ & $18.58 \pm 1.11^{* *}$ & $196.14 \pm 14.19$ \\
LSC-H & $219.44 \pm 10.70$ & $213.31 \pm 10.31$ & $21.49 \pm 1.48$ & $221.32 \pm 11.41$ \\
LSC-L & $235.24 \pm 13.08$ & $211.69 \pm 10.58$ & $20.00 \pm 1.39^{* *}$ & $204.36 \pm 14.31$ \\
LSD-H & $223.88 \pm 10.96$ & $212.51 \pm 13.61$ & $20.28 \pm 1.15^{*}$ & $202.58 \pm 10.65$ \\
LSD-L & $210.98 \pm 16.70$ & $227.27 \pm 17.80^{*}$ & $20.74 \pm 2.02$ & $182.02 \pm 16.32^{*}$ \\
\hline
\end{tabular}

The values are reported as mean \pm SD for 8 rats in each group. ${ }^{*} P<0.05$ and ${ }^{* *} P<0.01$ compared to the control.

diuretic activity of LS and its four fractions (LSA, LSB, LSC, and LSD) compared with that produced by furosemide in normal rats. After oral administration of LS and its four fractions, LS-H (458.0 mg/kg), LS-M (229.0 mg/kg), LSB-H
(323.6 mg/kg), and LSC-L $(8.8 \mathrm{mg} / \mathrm{kg})$ produced notable diuretic effects in comparison with the control group within $24 \mathrm{~h}$. It is important that the $323.6 \mathrm{mg} / \mathrm{kg}$ dose of LSB-H significantly increased the urine output volume comparable 
TABLE 7: Effects of LS, LSA, LSB, LSC, and LSD on the levels of Ang II, ANP, ADH, and ALD (pg/mL) in rat serum within $24 \mathrm{~h}$ after oral administration.

\begin{tabular}{lcccc}
\hline Group & Ang II & ANP & ADH & ALD \\
\hline Control & $219.43 \pm 16.19$ & $187.47 \pm 12.00$ & $23.09 \pm 1.12$ & $229.78 \pm 15.03$ \\
Furosemide & $218.53 \pm 15.99$ & $186.85 \pm 12.83$ & $20.31 \pm 1.70$ & $193.19 \pm 15.65$ \\
LS-H & $219.88 \pm 10.81$ & $217.13 \pm 14.22^{*}$ & $20.11 \pm 1.11$ & $232.80 \pm 19.79$ \\
LS-M & $213.24 \pm 10.54$ & $232.36 \pm 15.83$ & $23.70 \pm 1.83$ & $222.98 \pm 18.29$ \\
LS-L & $199.66 \pm 13.47$ & $219.78 \pm 11.54$ & $20.54 \pm 1.28$ & $202.21 \pm 19.17$ \\
LSA-H & $234.87 \pm 11.86$ & $193.99 \pm 9.21$ & $20.32 \pm 1.01$ & $231.86 \pm 14.03$ \\
LSA-L & $240.34 \pm 14.86$ & $231.18 \pm 13.70$ & $19.59 \pm 1.99$ & $171.40 \pm 5.83$ \\
LSB-H & $253.99 \pm 13.11$ & $224.45 \pm 17.83$ & $21.43 \pm 1.64$ & $201.52 \pm 14.85$ \\
LSB-L & $239.09 \pm 17.55$ & $202.17 \pm 19.57$ & $19.93 \pm 1.39$ & $216.85 \pm 17.01$ \\
LSC-H & $215.55 \pm 11.47$ & $211.94 \pm 3.83^{*}$ & $229.79 \pm 14.31$ \\
LSC-L & $235.24 \pm 12.85$ & $213.27 \pm 16.63$ & $17.09 \pm 0.66^{* *}$ & $209.30 \pm 18.69$ \\
LSD-H & $203.79 \pm 15.93$ & $205.46 \pm 14.23$ & $19.50 \pm 1.84^{*}$ & $200.22 \pm 8.46$ \\
LSD-L & $231.49 \pm 16.22$ & $206.85 \pm 19.71$ & $186.51 \pm 18.13^{*}$ \\
\hline
\end{tabular}

The values are reported as mean \pm SD for 8 rats in each group. ${ }^{*} P<0.05$ and ${ }^{* *} P<0.01$ compared to the control.

TABLE 8: Effects of LS, LSA, LSB, LSC, and LSD on TNF- $\alpha$ and IL-6 levels in urine and serum of rats within 24 hours after administration.

\begin{tabular}{lcccr}
\hline Group & TNF- $\alpha$ & Urine & & Serum \\
& $145.23 \pm 22.52$ & IL-6 & $143.26 \pm 28.06$ & IL-6 \\
\hline Control & $143.63 \pm 27.57$ & $72.98 \pm 13.99$ & $157.04 \pm 18.19$ & $72.33 \pm 14.42$ \\
Furosemide & $124.22 \pm 13.81$ & $74.28 \pm 15.85$ & $132.04 \pm 29.98$ & $73.00 \pm 13.80$ \\
LS-H & $133.71 \pm 10.65$ & $71.04 \pm 15.41$ & $139.90 \pm 26.89$ & $65.00 \pm 16.58$ \\
LS-M & $140.29 \pm 12.81$ & $78.68 \pm 16.32$ & $144.14 \pm 14.82$ & $68.20 \pm 13.81$ \\
LS-L & $129.00 \pm 16.04$ & $72.29 \pm 11.08$ & $135.28 \pm 30.58$ & $69.83 \pm 16.67$ \\
LSA-H & $150.42 \pm 21.77$ & $66.29 \pm 13.32$ & $125.14 \pm 12.81$ & $74.12 \pm 12.77$ \\
LSA-L & $142.03 \pm 15.04$ & $78.49 \pm 13.28$ & $152.62 \pm 28.04$ & $70.25 \pm 11.50$ \\
LSB-H & $133.51 \pm 10.80$ & $69.42 \pm 13.98$ & $139.21 \pm 31.27$ & $69.88 \pm 13.63$ \\
LSB-L & $131.41 \pm 21.70$ & $69.89 \pm 12.69$ & $146.63 \pm 24.64$ & $71.76 \pm 16.86$ \\
LSC-H & $130.78 \pm 19.43$ & $61.23 \pm 12.00$ & $150.14 \pm 14.18$ & $62.38 \pm 11.05$ \\
LSC-L & $146.98 \pm 23.31$ & $71.45 \pm 6.88$ & $139.26 \pm 21.28$ & $73.59 \pm 12.98$ \\
LSD-H & $140.21 \pm 19.62$ & $81.43 \pm 14.91$ & $134.78 \pm 32.01$ & $73.82 \pm 19.00$ \\
LSD-L & & & $75.81 \pm 15.07$ \\
\hline
\end{tabular}

The values are reported as mean \pm SD for 8 rats in each group.

to furosemide within $24 \mathrm{~h}$. Surprisingly, we determined that LSB has a dual effect on renal function, including promoting diuretic activity at a higher dose $(323.6 \mathrm{mg} / \mathrm{kg})$ and inhibiting diuretic activity at a lower dose $(80.9 \mathrm{mg} / \mathrm{kg})$, which is similar to other diuretics in TCM $[14,15]$. On the contrary, our results demonstrate a preliminary linear relationship between the LS and LSB doses and the diuretic biological response. Further research is underway to elucidate the dose-diuretic activity relationship of LS and its four fractions in normal rats. These results indicated that LS, LSB, and LSC present a notable diuretic effect, and the LSB dose should be given more attention in clinical applications.

$\mathrm{Na}^{+}$and $\mathrm{K}^{+}$were the major electrolytes located in the intracellular and extracellular fluids and play a regulatory role in maintaining body fluid homeostasis [17-19]. Our results showed that LS and its four fractions affect the urinary electrolytes and the acid-base alterations compared with the control group within $24 \mathrm{~h}$ in normal rats. A great variation in electrolyte excretion $\left(\mathrm{Na}^{+}\right.$and $\left.\mathrm{K}^{+}\right)$between promoting diuretic effects and inhibiting diuretic effects of LS and its four fractions as well as furosemide were observed. When promoting the urine output, LS, LSA, LSB, LSC, LSD, and furosemide produced similar increases in urine $\mathrm{Na}^{+}$ excretion but had slight effects on $\mathrm{K}^{+}$excretion. This increased $\mathrm{Na}^{+}$loss, which is due to the increase in the distal tubular $\mathrm{K}^{+}$concentration, and stimulated the ALD-sensitive potassium pump to increase the $\mathrm{K}^{+}$reabsorption in exchange for $\mathrm{Na}^{+}$and $\mathrm{H}^{+}$, which were excreted into the urine $[15,20]$. Although the LS and its four fractions affected the $\mathrm{Na}^{+}$and $\mathrm{K}^{+}$concentrations in urine, all drug groups cannot affect urine or serum $\mathrm{Na}^{+}-\mathrm{K}^{+}$-ATPase levels and urine $\mathrm{pH}$ values compared with the control group within $24 \mathrm{~h}$.

The RAAS is very important for body fluid regulation and plays a prominent role in controlling fluid homeostasis and maintaining electrolyte balance [18, 21, 22]. Moreover, the main three hormones in the RAAS including Ang II, $\mathrm{ADH}$, and ALD have been reported to decrease the glomerular filtration rate and increase tubular reabsorption of sodium ions [16-18]. ANP not only increases diuresis and $\mathrm{Na}^{+}$electrolyte excretion but also inhibits the activation of RAAS and blocks the release and/or actions of $\mathrm{ADH}$ and ALD [23-27]. On the contrary, ALD promotes urine $\mathrm{Na}^{+}$ and $\mathrm{H}_{2} \mathrm{O}$ reabsorption and regulates Ang II and ALD levels [18]. In addition, Ang II could also augment $\mathrm{Na}^{+}$ 
reabsorption and the release of $\mathrm{ADH}$. In this experiment, LS and its four fractions can suppress the RAAS, including ADH arrest by LSB ( 80.9 and $323.6 \mathrm{mg} / \mathrm{kg})$, LSC $(8.8 \mathrm{mg} / \mathrm{kg})$, and LSD (3.4 and $13.6 \mathrm{mg} / \mathrm{kg}$ ) and ALD arrest by LSD $(3.4 \mathrm{mg} / \mathrm{kg})$, as well as promote ANP release by LS $(229.0 \mathrm{mg} / \mathrm{kg})$, LSB $(323.6 \mathrm{mg} / \mathrm{kg})$, LSC $(35.2 \mathrm{mg} / \mathrm{kg})$, and LSD $(13.6 \mathrm{mg} / \mathrm{kg})$, while furosemide can suppress only arrest of $\mathrm{ADH}$ within $24 \mathrm{~h}$ compared with the control group. These results indicated that the diuretic effect of LS and its four fractions on normal rats is multichannel and multitarget including ANP, ADH, and ALD. Meanwhile, furosemide only affects the ADH level.

Therefore, we believe that LS and its active components are involved in the treatment of diuretic diseases with multiple components, multiple channels, and multiple targets. Although the diuretic and antidiuretic effects of these active components are not perfectly elucidated, previous phytochemical investigations suggested that diterpenoids, phenylethanoids, and flavonoids are their major components and exhibit various bioactivities [28-30]. Based on the above data, we hypothesized that these major bioactive components are responsible for the diuretic and antidiuretic activities, but much work still needs to be done.

Inflammation is a complex biological response to harmful stimuli, tissue injury, or infection and produced a number of proinflammatory mediators, such as TNF- $\alpha$ and IL-6 [31, 32]. Moreover, inflammatory cytokines, such as IL6 and TNF, are involved in the processes by which TCMs act on PBCRBS according to experimental researches and network pharmacology analysis [33-35]. On the contrary, the traditional Chinese medical concept of Huo Xue Li Shui (Chinese: 活血利水) refers to “PBCRBS" and “promoting blood circulation and diuresis" (PBCD), which excretes $\mathrm{H}_{2} \mathrm{O}$ out of the body in the form of urine [13]. Our previous study showed that the ethanol extract of L. supina showed a significant diuretic activity in TBS model rats, which are a rat model for the screening of PBCRBS and diuretic drugs [13]. For this reason, we also examined the levels of TNF- $\alpha$ and IL-6 in rats after treatment with LS and its four fractions (LSA-D) in this experiment. The ELISA results revealed that administration of LS and its four fractions did not affect the urine and serum TNF- $\alpha$ and IL- 6 levels in normal rats within $24 \mathrm{~h}$ in comparison with the control group. These results suggested that LS and its four fractions did not affect the innate immune system in normal rats.

\section{Conclusions}

To summarize our findings, this is the first reported notable diuretic effect of LS and its fractions (LSB and LSC) in normal rats, which probably inhibit the RAAS system and promote ANP release through multiple channels and multiple targets. We also first found that LSB has dual effects, namely, promoting and inhibiting diuretics in normal rats. Although the LS and its four fractions could change the urinary electrolyte concentrations $\left(\mathrm{Na}^{+}\right.$and $\left.\mathrm{K}^{+}\right)$, they did not affect the urine $\mathrm{pH}$ and urine and serum $\mathrm{Na}^{+}-\mathrm{K}^{+}$ATPase, TNF- $\alpha$, and IL- 6 levels within $24 \mathrm{~h}$ compared with the control group. This study will provide a quantitative basis for explaining the natural medicinal use of LS as a diuretic agent for edema and promoting the diuretic process. Further research is underway to elucidate the mechanism and determine the active components responsible for the diuretic and antidiuretic activities of LS.

\author{
Abbreviations \\ LS: $\quad$ The ethanol extract of Lagopsis supina \\ RAAS: Renin-angiotensin-aldosterone system \\ TCMs: Traditional Chinese medicines \\ PBCRBS: Promoting blood circulation and removing blood \\ stasis \\ TBS: $\quad$ Traumatic blood stasis \\ Ang II: Angiotensin II \\ ANP: Atriopeptin \\ ADH: Antidiuretic hormone \\ ALD: Aldosterone \\ HPLC: High-performance liquid chromatography \\ TLC: $\quad$ Thin layer chromatography \\ CMC- Sodium carboxymethyl cellulose \\ $\mathrm{Na}$ : \\ ELISA: Enzyme-linked immunosorbent assay \\ SD: $\quad$ Standard deviation \\ ANOVA: Analysis of variance \\ TNF: $\quad$ Tumor necrosis factor \\ IL-6: Interleukin-6.
}

\section{Data Availability}

The data used to support the findings of this study are available from the corresponding author upon request.

\section{Conflicts of Interest}

The authors declare that they have no conflicts of interest.

\section{Authors' Contributions}

Zhiyong Liu and Li Yang contributed equally to this work.

\section{Acknowledgments}

This work was financially supported by grants from the Jiangxi University of Traditional Chinese Medicine (nos. JXSYLXK-ZHYA0031，2016RC001， JXXT2017008, and JZYC19S48).

\section{References}

[1] L. Yang and J.-W. He, "Hosta plantaginea (Lam.) Aschers (Yuzan): an overview on its botany, traditional use, phytochemistry, quality control and pharmacology," RSC Advances, vol. 9, no. 60, pp. 35050-35058, 2019.

[2] J. He, X. Huang, Y. Wang et al., "A new flavonol glycoside from the flowers of Hosta plantaginea with cyclooxygenases1/2 inhibitory and antioxidant activities," Natural Product Research, vol. 33, no. 11, pp. 1599-1604, 2019.

[3] J.-W. He, L. Yang, Z.-Q. Mu et al., "Anti-inflammatory and antioxidant activities of flavonoids from the flowers of Hosta 
plantaginea," RSC Advances, vol. 8, no. 32, pp. 18175-18179, 2018.

[4] L. Yang, S.-T. Jiang, Q.-G. Zhou, G.-Y. Zhong, and J.-W. He, "Chemical constituents from the flower of hosta plantaginea with cyclooxygenases inhibition and antioxidant activities and their chemotaxonomic significance," Molecules, vol. 22, no. 11, p. 1825, 2017.

[5] P. L. Li, W. W. Su, S. Yun et al., "Toward a scientific understanding of the effectiveness, material basis and prescription compatibility of a Chinese herbal formula Dan-hong injection," Scientific Reports, vol. 7, p. 46266, 2017.

[6] State Administration of Traditional Chinese Medicine, Chinese Materia Medica, Vol. 8, Shanghai Science and Technology Press, Shanghai, China, 1999.

[7] M. R. Jia and Y. Zhang, Dictionary of Chinese Ethnic Medicine, p. 468, China Medical Science Press, Beijing, China, 2016.

[8] M. Bai, D. Liu, X. Yan, and M. Miao, Chinese Journal of Modern Applied Pharmacy, vol. 31, pp. 517-522, 2014.

[9] C. Y. Niu, J. C. Li, Y. K. Liu, and Z. G. Zhao, Chinese Pharmaceutical Journal, vol. 41, pp. 914-917, 2006.

[10] L. M. Zhang, H. Jiang, Y. K. Liu, X. F. Zhang, C. Y. Niu, and W. M. Zhang, Journal of Chinese Medicinal Materials, vol. 27, pp. 509-511, 2004.

[11] H. F. Liang, W. P. Wang, Y. P. Zhang, S. T. Du, and H. Jiang, Lishizhen Medicine and Materia Medica Research, vol. 19, pp. 1650-1651, 2008.

[12] Y. C. Zhang, R. Han, Y. L. Hou et al., Lishizhen Medicine and Materia Medica Research, vol. 19, pp. 1909-1910, 2008.

[13] J. He, L. Zeng, R. Wei et al., "Lagopsis supina exerts its diuretic effect via inhibition of aquaporin-1, 2 and 3 expression in a rat model of traumatic blood stasis," Journal of Ethnopharmacology, vol. 231, pp. 446-452, 2019.

[14] D.-Q. Chen, Y.-L. Feng, T. Tian et al., "Diuretic and antidiuretic activities of fractions of Alismatis rhizoma," Journal of Ethnopharmacology, vol. 157, pp. 114-118, 2014.

[15] Y.-L. Feng, H. Chen, T. Tian, D.-Q. Chen, Y.-Y. Zhao, and R.-C. Lin, "Diuretic and anti-diuretic activities of the ethanol and aqueous extracts of Alismatis rhizoma," Journal of Ethnopharmacology, vol. 154, no. 2, pp. 386-390, 2014.

[16] J. G. Yu, J. M. Guo, K. Y. Zhu et al., Scientific Reports, vol. 7, p. $3828,2017$.

[17] K. Kumar, S. Sharma, V. Vashishtha et al., "Terminalia arjuna bark extract improves diuresis and attenuates acute hypobaric hypoxia induced cerebral vascular leakage," Journal of Ethnopharmacology, vol. 180, pp. 43-53, 2016.

[18] J.-W. Lou, L.-L. Cao, Q. Zhang et al., "The toxicity and efficacy evaluation of different fractions of Kansui fry-baked with vinegar on Walker-256 tumor-bearing malignant ascites effusion rats and normal rats," Journal of Ethnopharmacology, vol. 219, pp. 257-268, 2018.

[19] J. Terry, Journal of Intravenous Nursing, vol. 17, pp. 240-247, 1994.

[20] S. Kardasz, "The function of the nephron and the formation of urine," Anaesthesia \& Intensive Care Medicine, vol. 10, no. 6, pp. 265-270, 2009.

[21] G. Remuzzi, N. Perico, M. Macia, and P. Ruggenenti, "The role of renin-angiotensin-aldosterone system in the progression of chronic kidney disease," Kidney International, vol. 68, pp. 57-65, 2005.

[22] B. Xue, Z. Zhang, C. F. Roncari, F. Guo, and A. K. Johnson, "Aldosterone acting through the central nervous system sensitizes angiotensin II-induced hypertension," Hypertension, vol. 60, no. 4, pp. 1023-1030, 2012.
[23] J. D. Baxter, J. A. Lewicki, and D. G. Gardner, "Atrial natriuretic peptide," Nature Biotechnology, vol. 6, no. 5, pp. 529-546, 1988.

[24] C. I. Johnston, G. P. Hodsman, R. W. Harrison, F. A. O. Mendelsohn, and K. Tsunoda, "Regulation of cardiac preload by atrial natriuretic peptide in congestive cardiac failure," The American Journal of Medicine, vol. 84, no. 3, pp. 105-111, 1988.

[25] J. H. Laragh, The New England Journal of Medicine, vol. 313, pp. 1330-1340, 1985.

[26] K. Nakayama, H. Ohkubo, T. Hirose, S. Inayama, and S. Nakanishi, "mRNA sequence for human cardiodilatinatrial natriuretic factor precursor and regulation of precursor mRNA in rat atria," Nature, vol. 310, no. 5979, pp. 699-701, 1984.

[27] H. Yasue, K. Obata, K. Okumura et al., "Increased secretion of atrial natriuretic polypeptide from the left ventricle in patients with dilated cardiomyopathy," Journal of Clinical Investigation, vol. 83, no. 1, pp. 46-51, 1989.

[28] H. Li, M.-M. Li, X.-Q. Su et al., "Anti-inflammatory labdane diterpenoids from Lagopsis supina," Journal of Natural Products, vol. 77, no. 4, pp. 1047-1053, 2014.

[29] J. Zhang, Z. Huang, H.-X. Huo et al., "Chemical constituents from Lagopsis supina (steph.) Ik.-Gal. Ex knorr,” Biochemical Systematics and Ecology, vol. 61, pp. 424-428, 2015.

[30] J. Zhang, D. R. Pang, Z. Huang et al., China Journal of Chinese Materia Medicine, vol. 40, pp. 3224-3228, 2015.

[31] J. M. Han, E. K. Lee, S. Y. Gong, J. K. Sohng, Y. J. Kang, and H. J. Jung, "Sparassis crispa exerts anti-inflammatory activity via suppression of TLR-mediated NF- $\kappa$ B and MAPK signaling pathways in LPS-induced RAW264.7 macrophage cells," Journal of Ethnopharmacology, vol. 231, pp. 10-18, 2019.

[32] Y.-H. Zou, L. Zhao, Y.-K. Xu et al., "Anti-inflammatory sesquiterpenoids from the Traditional Chinese Medicine Salvia plebeia: regulates pro-inflammatory mediators through inhibition of NF- $\kappa \mathrm{B}$ and Erk1/2 signaling pathways in LPSinduced Raw264.7 cells," Journal of Ethnopharmacology, vol. 210, pp. 95-106, 2018.

[33] X. Dang, J.-J. Miao, A.-Q. Chen et al., "The antithrombotic effect of RSNK in blood-stasis model rats," Journal of Ethnopharmacology, vol. 173, pp. 266-272, 2015.

[34] M. Lv, T. Y. Wang, X. X. Tian et al., Acta Pharmaceutical, vol. 50, pp. 1135-1141, 2015.

[35] G. H. Song, Q. M. Zhang, B. Z. Pang et al., Journal of Traditional Chinese Medicine, vol. 35, pp. 211-217, 2015. 

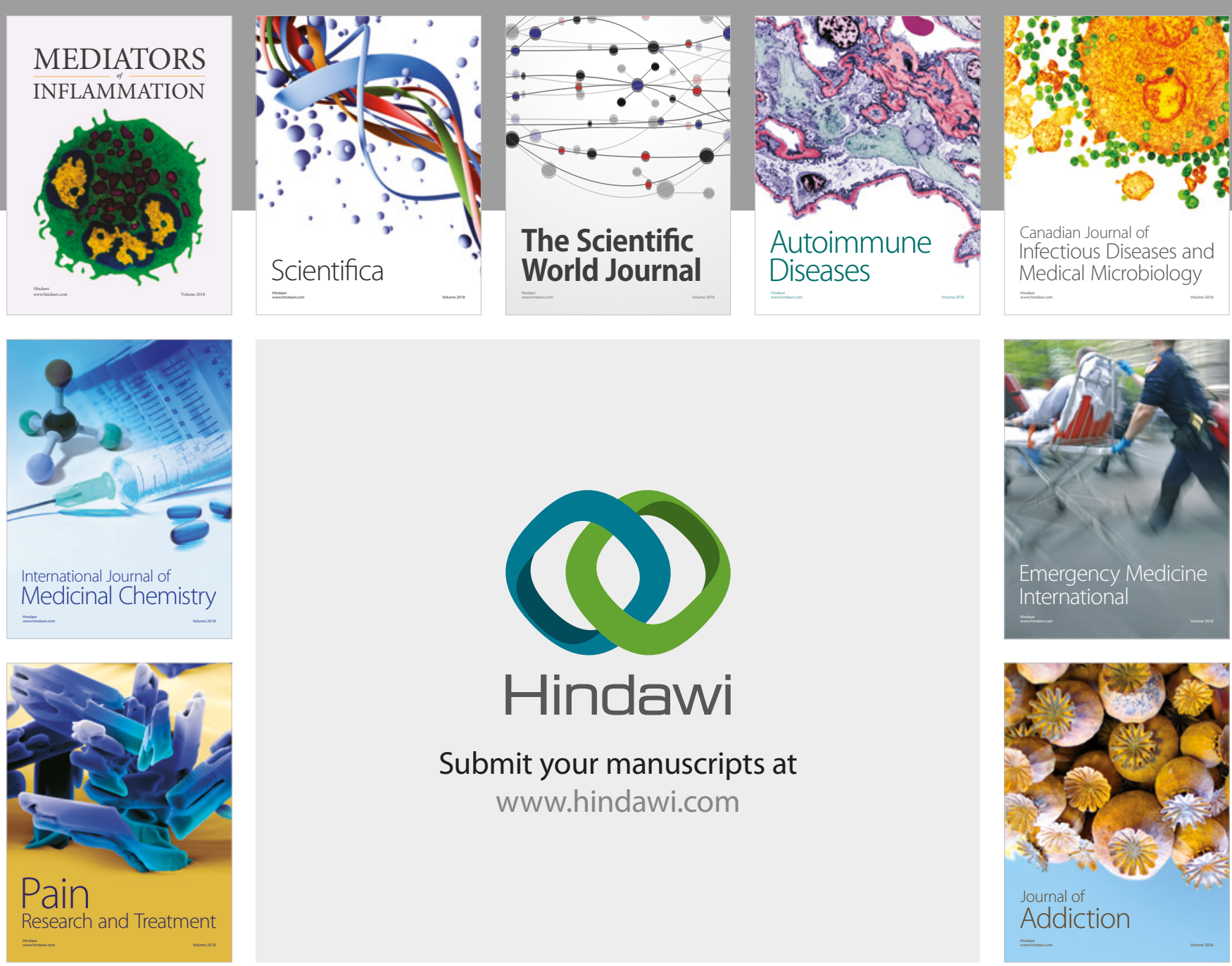

Canadian Journal of
Infectious Diseases and Medical Microbiology

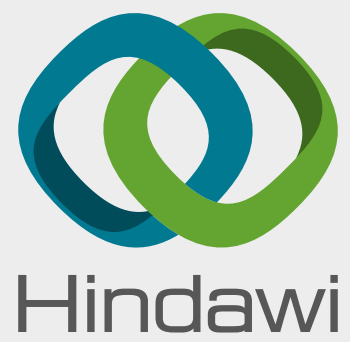

Submit your manuscripts at

www.hindawi.com
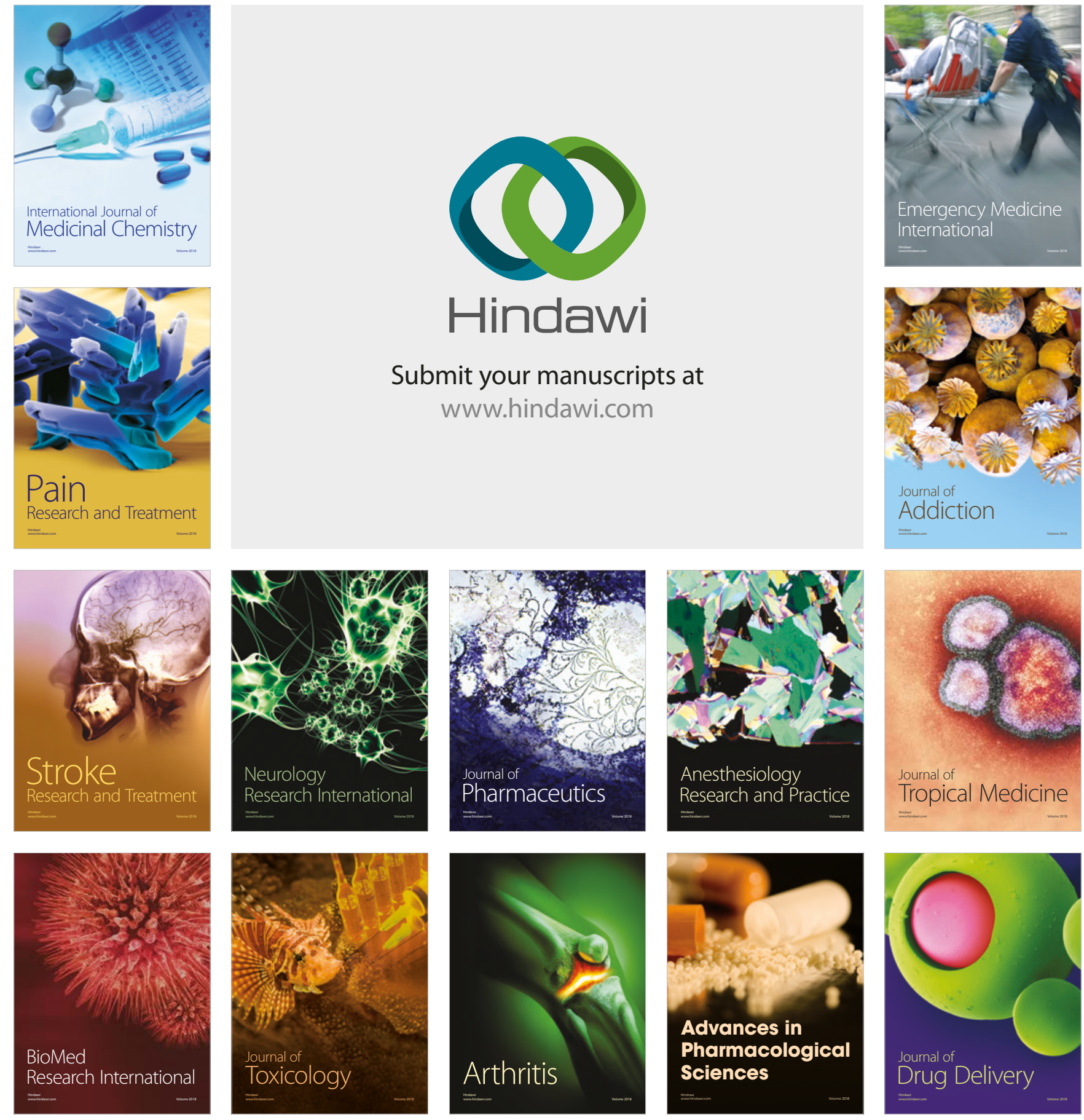